\title{
TRACKING IMPLICIT TRAJECTORIES
}

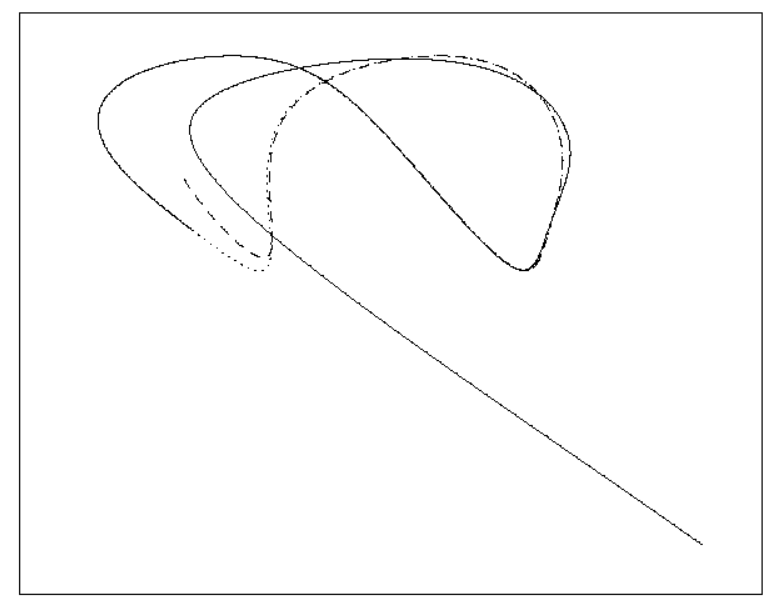

Neil H. Getz and Jerrold Fi. Marsden

Memorandum No. CPAM 629)

17 February 1995

\section{CENTER FOR PURE AND APPLIED MATHEMATICS}

Department of Mathematics

University of Califormia at Berkeley

Berkeley, CA 94720 



\title{
Tracking Implicit Trajectorics*
}

\author{
Neil H. Getz \\ Electrical Engineering and Computer Sciences \\ University of California at Berkeley \\ Berkeley, California 94720 \\ getz@eecs, berkeley , edu \\ Jerrold F. Marsden \\ Control and Dynamical Systems \\ California Institute of T'cchnology \\ Pasadena, California 91125 \\ marsden@cds.caltech. edu \\ February 17, 1995
}

\begin{abstract}
Output trasking of implesily delined reference trajectorises is cxamined. A continuous-time nonlinear dynamical system is constructed that produces explicit estimates of time-varying implicit trajectories. We prove that incorporation of this "dynamic inverter" into a tracking controller provides exponential output trasking of the implicitly defined t.rajectory for nonlinear control systems having vector relative degree and well-behaved internal dynanmics.
\end{abstract}

Key Words. dynamic, inversion, implicit, tracking, inverse kinematic problem. nonlinear control, robot control, singular perturbations

*Presented at IFAC Symposium on Nonlinear Control System Design, 'lahoe Cily, June $26-28,1995$. 
'Tracking Implicit Trajectories

\section{Introduction}

In this article we will consider the problen of output tracking where the refcrence output which we wish to track is definod implicitly. Wc will roly upon a. continuous time dynamical technique for inverting nonlinear maps, and will refer to this technique as dynamic inversion [CM94]. We will join dynanic inversion to a tracking controller in order to provide an explicit estimator for the reference outpul.

We will first give a bricf review of the cssential clements of dynamic inversion. Dynamic inversion is then incorporated into a tracking controller for tracking of the implicit reference trajectory. An example of output tracking for a simple robot arm illustrates application of the theory.

\section{Dynamic Inversion}

Iynamic inversion is a methodology for using continuous time dynamics to provide an estimale of time-varying roots of time dependent maps. The methodology also provides a framowork in which to vicw and gencralize certain clements of extant dynamical methods for inverting nonlinear maps using for instance gradicnt flows, ncural networks, and the tochniques of [NTV91]. In dynamic inversion one associates with a map $f^{\prime}(\theta, t)$ a dynamical system $\dot{\theta}=\Phi(\theta, t)$ with the crucial property that an isolated root $\theta_{\infty}(l)$ is exponentially attractive. Dynamic inversion depends intimatcly upon the notion of a dynamic inverse which we now define.

Definition 1 Lct $F: \mathbb{P}^{n} \times \widehat{Z}_{+} \rightarrow \mathbb{P}^{n} ;(\theta, t) \mapsto F(\theta, t)$ bc continuous in $\theta$ and pitcewise conlinuous in $l$. Lel $O_{*}(l)$ be a conlinuous isolaled solulion of $\Gamma(0, l)=$ 0 . $A \operatorname{map} G: \mathbb{R}^{n} \times \mathbb{R}_{+} \rightarrow \mathbb{P}^{n} ;(w, t) \mapsto G_{T}(w, t)$ is called a dynamic inverse of $H^{\prime}$ on the ball $\mathcal{B}_{r}:=\left\{z \in \mathbb{R}^{\prime \prime} \mid\|z\| \leq r\right\}, r>0$, if $(1)$ the map $G\left(H^{\prime}(\theta, t), t\right)$ is Lipschitz in $w$ and piecewise continuous in $l$, and (2) lhere is a fixed real number $\beta$, with $0<\beta<\infty$, such that

$$
z^{T} G\left(F\left(z+\theta_{\infty}(l), l\right), l\right) \geq \beta\|z\|_{2}^{2}
$$

for all $z \in \mathcal{B}_{r}$.

Remark 1 If $G(w, t)$ is a dynamic inverse of $F(\theta, t)$ with constant $\beta$, then for any $\mu \in K_{+} \mu G(u, t)$ is a dynamic inverse of $\digamma^{\prime}(\theta, t)$ with constant $\mu \beta$.

Sufficient conditions for the cxistence of a dynamic inverse for all $t \in Z+$ arc mild as shown by the following lemma, proof of which may be found in [GM94].

Lemma 1 Let $\theta_{x}(t)$ bo continuous isolated solution of $F(\theta, t)=0$. Assume $F(0, l)$ is $C^{2}$ in 0 and piecewise conlinuous in $l$. Lel $D_{1} F\left(\theta_{\times}(l), l\right)$ be nonsingular for all $t$. Let $D_{1} F\left(\theta_{x}(t), t\right)$ and $D_{1} F\left(\theta_{*}(t), t\right)^{-1}$ be bounded uniformly 
in $t$. For all $z \in \mathcal{B}_{r}$, let $D_{1}^{2} F\left(z+\theta_{*}(t), t\right)$ be bounded uniformly in $t$. Under these conditions, for any particular time $t_{1} \geq 0$, there exists an interval $\left[\ell_{0}, \ell_{2}\right] \subset \mathcal{Z}_{+}$wilh $l_{0}<\ell_{1}<l_{2}$, and a ball $\mathcal{B}_{r}$ such that for any parlicular $\theta_{p} \in \mathcal{B}_{r}, G(w, t):=D_{1} F\left(\theta_{p}, t_{1}\right)^{-1}, w$ is a dynamic inverse of $F(\theta, t)$ on $\mathcal{B}_{r}$ for all $l \in\left[l_{0}, l_{2}\right]$.

An important special class of dynanic inverses is the class in which $G$ is of the form $G(w, \theta, t)$, where $\theta$ is the solution of a dynamical system which estimates $\theta_{*}$. 'Thus $G$ inherits part of its time dependence from its $\theta$-dependence. In this casc wc say that $G(w, \theta, t)$ is a state dependent dynamic inverse of $F$ on $\mathcal{B}_{r}$ if

$$
z^{T} G\left(F\left(z+\theta_{*}(t), t\right), z+\theta_{*}(t), t\right) \geq \beta\|z\|_{2}^{2}
$$

for all $z \in \mathcal{B}_{r}$.

The dynamic inversion theorcm below tics the dynamic inverse to dynamical estimation of a continuous isolated solution of $H^{\prime}(\theta, t)=0$. This theorem is proven in [GM94].

Theorem 1 (Dynamic Inversion Theorem) Let $\theta_{x}(t)$ be a continuous isolated solution of $F^{\prime}(\theta, t)=0$, with $F^{r}: K^{n} \times \mathbb{R}_{+} \rightarrow \mathbb{R}^{n *} ;(\theta, t) \mapsto F(\theta, t)$. Assume that $G: \mathbb{R}^{n} \times \mathbb{R}^{n} \times \mathbb{E}_{+} \rightarrow \mathbb{Z}^{n} ;(u, 0, l) \mapsto C(w, 0, l)$, is a slalt-dependenl dynamic inverse of $F(\theta, t)$ on $\mathcal{B}_{r}$, for some finite $\beta>0$. Let $E: \mathbb{P}^{n} \times \mathbb{E}_{+} \rightarrow \mathbb{Z}^{n}$; $(\theta, t) \mapsto f^{\prime}(\theta, t)$ be locally Lipshits in $\theta$ and piecewise continuous in $t$. Assume. that for some fixtd $\kappa \in(0, \infty), E(0, l)$ salisfies

$$
\left\|E\left(z+0_{*}(l), l\right)-\dot{0}_{*}(l)\right\|_{2} \leq \kappa\|z\|_{2}
$$

for all $z \in \mathcal{B}_{r}$. Let $\theta(t)$ denote the solution to the system.

$$
\dot{\theta}=-\mu G(F(\theta, t), \theta, t)+F(\theta, t)
$$

with initial condition $\theta(0)$ satisfying $\theta(0)-\theta_{*}(0) \in \mathcal{B}_{r}$. Then

$$
\left\|O(l)-O_{*}(l)\right\|_{2} \leq\left\|0(0)-O_{*}(0)\right\|_{2} e^{-\left(\psi / \beta-n_{0}\right) t}
$$

for all $l \in \mathbb{E}_{+}$, and in particular if $\mu>\kappa / \beta$, then $\theta(l)$ converges to $O_{\times}(l)$ cxponcntially.

The map $F(\theta, t)$ in 'I'heorem 1 is usually chosen to be a $\theta$ - and $t$-dependent estimator for $\dot{\theta}_{*}$. We will show one way of constructing such an estimator below.

Though a dynamic inverse need not be linear, a linear one is often easy to obtain as indicated by Lemma 1 . Let $G(u, \theta, t):=D_{1} F^{\prime}(\theta, t)^{-1} \cdot w$. It follows from Lemma 1 and Theorem 1 that if $\mu$ is sufficiently large, $\left\|\theta(0)-\theta_{*}(0)\right\|$ is sufficicntly small, and $G(w, \theta(0), 0)$ is a dynamic inverse of $F(\theta, t)$ around $t=0$, then $G(u, 0, \ell)$ is a dynanic inverse of $F(0, \ell)$ for all $l>0$. Example 1 will illustrate application of Lcmma 1 and Theorcm 1 to the cstimation of $\theta_{x}(t)$. 
Example 1 Assume that the assumptions of Lemma 1 hold. We may obtain an estimator $\xi^{\prime}(\theta, t)$ for $\dot{\theta}_{*}$ by differentiating $H^{\prime}\left(\theta_{*}(t), t\right)=0$,

$$
D_{1} F\left(\theta_{*}(t), t\right) \dot{\theta}_{*}(t)+D D_{2} F^{\prime}\left(\theta_{*}, t\right)=0,
$$

solving for $\dot{\theta}_{*}$, and replacing $\theta_{x}$ by $\theta$ to get

$$
E(0, \ell):=-D_{1} \Gamma(0, \ell)^{-1} D_{2} \Gamma(0, \ell) .
$$

Assume that $r$ has been chosen sufficiently small, and that $D_{2} f^{\prime}(\theta, t)$ is sufficiently bounded so that $E(\theta, t)$ satisfics (3) for all $z \in \mathcal{B}_{r}$. Let

$$
C(w, 0, \iota):=D_{1} \Gamma(0, \ell)^{-1} \cdot w
$$

and assume that $r$ is small enough that $G$ is a dynamic inverse of $F$ on $\mathcal{B}_{r}$. If $\left(\theta(0)-\theta_{*}(0)\right) \in \mathcal{B}_{r}$, and $\mu$ is sufficiently large, then by Theorem 1 the approximation error $z(l):=0(l)-0_{*}(l)$ using (1) will decay exponentially to zero. $\triangle$

Example 2 shows how one may invert a time-varying matrix dynamically. See [GM95] for a more comprehensive handling of dynamic matrix inversion as well as polar decomposition.

Example 2 Consider the problem of estimating the inverse $\Gamma_{*}$ of a time-dependent matrix $A(t)$. Assume that $\Lambda(t)$ is $C^{1}$ in $t$ and nonsingular.

In order for $l_{\times}$to be the inverse of $A(t), l_{\times}$must satisfy $A(t) I^{\prime}-I=0$. Accordingly we let

$$
\Gamma^{M}(\Gamma, l):=A(l) \Gamma-I .
$$

We may obtain an estimator $H^{M}\left(I^{\prime}, t\right)$ for $\dot{I}_{*}^{\prime}$ by differentiating $A(t) I_{*}^{\prime}-l=0$ with respect to $\ell$, solving for $\dot{\Gamma}_{*}$, and replacing all occurrences of $\Gamma_{*}$ by $\Gamma$ to get

$$
E^{M}(\Gamma, l)=-\Gamma \dot{A}(l) \Gamma .
$$

For a dynamic inverse consider $\left(D_{1} F^{M}(\Gamma, t)\right)^{-1} \cdot w$. Differentiating $F^{M}$ with respect to $\Gamma$ gives

$$
D_{1} F^{\prime M}(i, t)=A(t)
$$

whose inverse is $I_{*}^{*}$. So a choice of dynamic inverse is

$$
G^{M}(u, l)=\ell \cdot w
$$

for $I^{\prime}$ sufficiently close to $I_{*}^{\prime}$. 'T'he dynamic inverter for this problem then takes the form

$$
\begin{aligned}
\dot{\Gamma} & =-\mu G^{M}\left(F^{M}(\Gamma, t), \Gamma\right)+E^{M}(\Gamma, t) \\
& =-\mu I(A(t) l-I)-I \dot{A}(t) I^{\prime}
\end{aligned}
$$


and we choose as initial conditions $\Gamma(0)$ sufficicntly close to $A^{-1}(0)$. Theorcm 1 guarantees that for sufficiently large $\mu$, equation (13) will produce an estimator $\Gamma$ which converges exponentially to $\Gamma_{*}$ at a rate determined by our choice of $\mu$. $\triangle$

We may now call upon insights from Example 2 in order to obtain a dynamic inverse dynamically while simultaneously using that dynamic inverse to estimate $0_{*}(l)$.

Example 3 Suppose that we wish to solve $F(0, l)=0,0 \in \mathbb{R}^{n}$. Let the assumptions of Lemma 1 hold. Wo wish to cstimate $\theta_{*}$ whilc providing a timedependent linear dynamic inverse of $F(\theta, t)$ based on knowledge of $\omega_{1} F^{\prime}(\theta, t)$. Assume that we have a representation of $D_{1} F(0, l)$ that is $C^{2}$ in 0 and $C^{1}$ in $t$. Let $I$ ' denote our estimator for $D_{1} F(\theta, t)^{-1}$. We may then estimate $\dot{0}_{*}(l)$ as follows: Differentiate $F\left(\theta_{x}, l\right)=0$, solve for $\dot{\theta}_{*}$ : and substitute $\Gamma$ for $D_{1} F\left(\theta_{*}(t), t\right)^{-1}$ and $\theta$ for $\theta_{x}$ to obtain an cstimator for $\dot{\theta}_{*}$ in torms of $\Gamma$ : $\theta$, and $t$ :

$$
E(\Gamma, 0, \ell):=-\Gamma D_{2} \Gamma(0, \ell)
$$

Assume that $E(\Gamma, \theta, t)$ is $C^{-1}$ in its arguments. Using $E(\Gamma, \theta, t)=\left[E_{i}(\Gamma, \theta, t)\right]_{i \in \underline{n}}$, by (10) we may estimate $\dot{\Gamma}_{*}$ with

$$
E^{M}(\Gamma, 0, \iota):=-\left.\Gamma \frac{d}{d t} D_{1} F(0, \iota)\right|_{\dot{\theta}=L(\Lambda, \theta, t)} \Gamma
$$

where

$$
\left.\frac{d}{d t} D_{1} \Gamma(\theta, l)\right|_{\dot{\theta}=L^{\prime}\left(I^{\prime} ; \theta, t\right)}:=\sum_{i=1}^{n} \frac{\partial D_{1} F(\theta, t)}{\partial \theta_{i}} E_{i}(\Gamma, 0, l)+\frac{\partial \nu_{1} F^{\prime}(\theta, t)}{\partial t}
$$

In this casc

$$
F^{M}(\Gamma, \theta, t):=D_{1} F(\theta, t) \Gamma-I
$$

Let $G^{M}(w, \Gamma):=\Gamma \cdot w$ as in Example 2.Theorem 1 now lells us that we may estimate $\theta_{*}(t)$ with the system of coupled nonlincar differential cquations

$$
\left[\begin{array}{l}
\dot{l} \\
\dot{\theta}
\end{array}\right]=-\mu\left[\begin{array}{ll}
I^{\prime} & 0 \\
0 & \Gamma
\end{array}\right] \cdot\left[\begin{array}{c}
\nu_{1} F^{\prime}(\theta, t) l-l \\
\Gamma(0, l)
\end{array}\right]+\left[\begin{array}{c}
F^{M}(l ; \theta, t) \\
-\Gamma D_{2} \Gamma(0, l)
\end{array}\right]
$$

with guaranteed exponential convergence of $(I ; \theta)$ to $\left(I_{*}^{\prime}, \theta_{\infty}\right)$. 


\section{Tracking Implicit Trajectories}

We now apply dynamic inversion to the problem of output tracking where the reference signal is defined implicitly. The problem we wish to solve is this: Find an input $u$ to a control system such that for all initial states in some ball about the origin, the output $y$ of the dynamical system converges to a desired implicitly defined output function $\theta_{*}(t)$. For simplicity we will assume that the nonlinear system we control hats the same number of inputs as outputs.

Let $\underline{k}:=\{1,2, \ldots, k\}$. Consider the following nonlinear control system:

$$
\left\{\begin{aligned}
\dot{\xi}_{j}^{i} & =\xi_{j+1}^{i}, \quad i \in \underline{m}, j \in \underline{r_{m}-1} \\
\dot{\xi_{r}} & =u \\
\dot{\eta} & =f(\xi, \eta, u, t) \\
y_{i} & =\xi_{1}^{i}, \quad i \in \underline{m}
\end{aligned}\right.
$$

with $\xi_{i}^{i} \in \mathbb{K}$, where $\xi_{r}:=\left[\xi_{1}, \ldots, \xi_{1}^{m^{m}}\right]^{T} \in \mathbb{R}^{\mathbb{N}^{m}}$. Let $p:=r_{1}+\ldots+r_{m}$, with $\xi \in \mathbb{R}^{p}, \eta \in \mathbb{R}^{n-p}$, inpul $u \in \mathbb{Z}^{m}$, oulpul $y \in \mathbb{Z}^{m}$. Assume $\int$ is a smoollı $\mathbb{H}^{p}$ valucd function of $\xi, \eta, u$, and $t$. Multi-input, multi-output systems having wclldefined vector relative degree $\left[r_{1}, \ldots, r_{r i}\right]$ may be put into the form 17 through a statc-dependent change of coordinates [Isi89]. We refer to the evolution of $\eta$ as the internal dynamics of (17).

Let $y_{d t}(l) \in \mathbb{Z}^{m}$ salisly $y_{d i}(l) \in C^{r_{*}-1}$. Lel

$$
\left\|y_{d}\right\|_{Y}:=\max _{i \in \underline{\underline{m l}}} \sup _{t \in \mathbb{R}_{+}}\left\{\left|y_{d i}^{(0)}(t)\right|, \ldots,\left|y_{d i}^{\left(r_{i}\right)}(t)\right|\right\} \text {. }
$$

Let $\mathcal{B}_{k}^{Y}$ be the open $k$-ball in the $\|\cdot\|_{Y}$ norm. Assume that if output $y \in \mathcal{B}_{k}^{Y}$ : implies $\|\eta\|$ is bounded.

Let $\Gamma: \mathbb{R}^{m} \times \mathbb{E}_{+} \rightarrow \mathbb{R}^{m}$ be such that $O_{*}(l) \in \mathcal{B}_{r,}^{Y}$ is a continuous isolated solution of $F(\theta, t)=0$. Assume that $F(\theta, t)$ is smooth in $\theta$ and $t$. Let $\left\{\beta_{i}\right\}, i \in$ $\{0, \ldots, r-1\}$ be chosen to be the cocfficients of the polynomial $s^{r}+\sum_{i=0}^{r-1} \beta_{i} s^{i}$ such that all roots of the polynomial have strictly negative real parts. If we had direct access to $\theta_{x}^{(i)}, i \in\{0, \ldots, r\}$, where $\theta_{*}^{(k)}$ denotes the $k^{\text {th }}$ derivative of $0_{*}$ with respect to time, and $\theta_{*}^{(0)}:=0_{*}$, then the choice of input $u_{i}=\theta_{x}^{\left(r_{i}\right)}-$ $\sum_{k=1}^{r_{k}} \beta_{k}\left(\xi_{k}^{i}-\theta_{*}^{(k-1)}\right), i \in \underline{m}$ would cause $y$ to track $\theta_{x}$ with exponentially decaying error.

Wo will join the dynamic inverter of Example 3 to the control system (17) using singular perturbation theory to prove stability of the combination.

Let $E^{0}:=0$. In a similar manner to the manner in which $E^{1}(\Gamma, 0, \ell)$ was obtained, we may obtain an estimator for $\theta_{*}^{(k)}$ for any $k \geq 1$ by the following recursive procedure: (1) Differentiatc $\left(d^{k-1} / d t^{k-1}\right)\left(F\left(\theta_{x}, t\right)=0\right)$ with respect to $\ell$. (2) Replace $\Gamma_{*}, \theta_{\infty}$ and $\theta_{*}^{(k-1)}$ by their estimators $\Gamma, 0$, and $E^{k-1}(\Gamma, 0, \ell)$ respectively. 
Consider the dynamic inverter

$$
\left[\begin{array}{c}
\dot{I} \\
\dot{\theta}
\end{array}\right]=-\mu \tilde{G}\left(\hat{H}\left(I^{\prime}, \theta, t\right), I^{\prime}\right)+\tilde{H}\left(I^{\prime}, \theta, t\right),
$$

where

$$
\begin{gathered}
\tilde{F}(\Gamma, \theta, t)=\left[\begin{array}{c}
D_{1} \Gamma(\theta, l) \Gamma-I \\
F(\theta, t)
\end{array}\right]: \dot{G}\left(w^{M}, w, \Gamma\right)=\left[\begin{array}{c}
\Gamma \cdot w^{M} \\
\Gamma \cdot w
\end{array}\right], \\
\hat{E}^{1}(\Gamma, \theta, \ell)=\left[\begin{array}{c}
E^{M}(\Gamma, \theta, t) \\
H^{-1}(l, \theta, t)
\end{array}\right],
\end{gathered}
$$

with $E^{M}(\Gamma, 0, l)$ defined as in (15). The following theorem asserts that the concatenation of the dynamic inverter (18) with the control systcm (17) can be used for exponentially convergent tracking of implicit trajectories.

Theorem 2 (Implicit Tracking Theorem) Lct

$$
u_{i}=E_{i}^{r_{i}}(\Gamma, 0, \ell)-\sum_{k=1}^{r_{i}} \beta_{k}\left(\xi_{k_{i}}^{i}-E_{i}^{k-1}(\Gamma, 0, \ell)\right)
$$

for $i \in \underline{m}$, where $E^{0}=0$, and $E^{j}, j \in \underline{m}$ are defined as above, and whert 0 and $\Gamma$ are the solutions to $(18)$. If $\left(\theta(0)-\theta_{*}(0)\right),\left(\Gamma(0)-\Gamma_{*}(0)\right)$, and $\left(\xi_{j}^{i}(0)-\theta_{x_{i}}^{(j-1)}(0)\right)$, $i \in \underline{m}, j \in \underline{r_{i}}$, are sufficiently small, then $y(t)$, the output of $(17)$, converges cxponcntially to $\theta_{*}(t)$.

Proof: First note that if $0(l) \equiv 0_{*}(l) \in \mathcal{B}_{*}^{Y}$ then

$$
u_{i}=\theta_{*}^{\left(r_{i}\right)}-\sum_{k=1}^{r_{k}-1} \beta_{k}\left(\xi_{k}^{i}-\theta_{\infty}^{\left(r_{i}\right)}\right), i \in \underline{m}
$$

and (17) has exponentially stable error tracking dynamics with $\|\eta\|$ bounded. Second, by Theorem 1. (18) has exponentially stable estimation error dymanics for $\epsilon$ sufficiently small. 'l'hird, let $\epsilon:=1 / \mu$ so that the dynamic inverter becomes

$$
c\left[\begin{array}{c}
\dot{I} \\
\dot{\theta}
\end{array}\right]=-\tilde{G}(\tilde{F}(\Gamma, \theta, t), \Gamma)+c \tilde{E}(\Gamma, \theta, t) \text {. }
$$

When $\epsilon=0$,

$$
0=-\tilde{G}\left(\tilde{h}(I, \theta, t), I^{\prime}\right)+0
$$

which implies that $(\Gamma, 0)=\left(\Gamma_{*}, 0_{*}\right)$. Thus, if $\|\eta\|$ is bounded under application of (19), then by Thoorem 8.3 of [Kha92] regarding exponential stablility of 


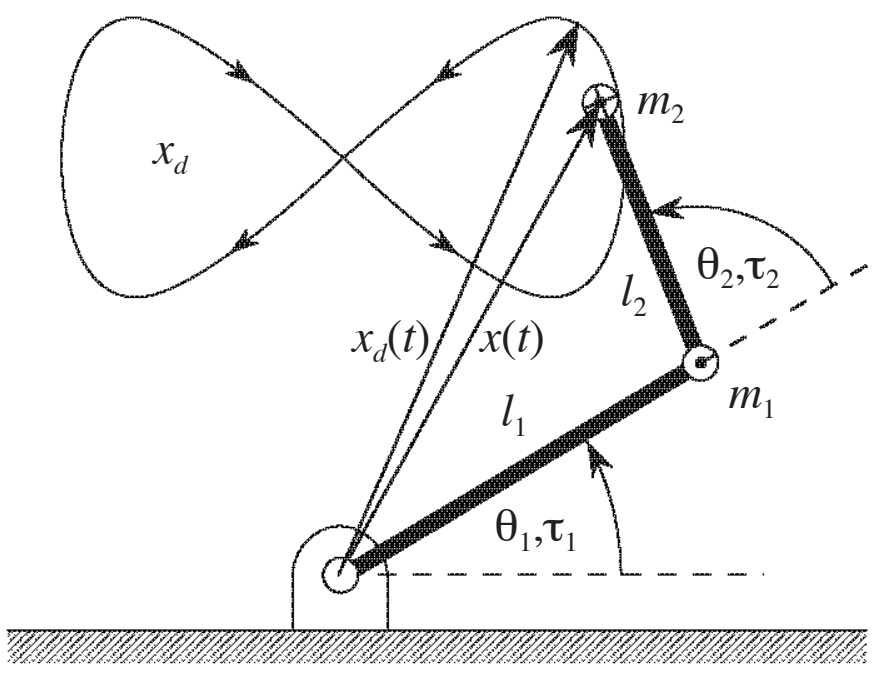

Figure 1: $A$ two-link robot arm with joint angles $\theta=\left(\theta_{1}, \theta_{2}\right)$, joint torques $\tau=\left(\tau_{1}, \tau_{2}\right)$, end-eflector position $x$, desired end-eflector position $x_{d}$, link lengths $l_{1}$ and $l_{2}$, and link masises $m_{1}$ and $m_{2}$.

singularly perturbed systems: $\xi_{j}^{i}(t) \rightarrow \theta_{* i}^{(j-1)}(t)$ exponentially for all $i \in \underline{m}$ : $j \in r_{i}$, and $\epsilon$ sufficiently small. Consequently: $y(l) \rightarrow 0_{*}(l)$ exponentially.

Boundedncss of $\|\eta\|$ requires only that $\left\|y(t)-\theta_{x}(t)\right\|$ be sufficiently small, but because the exponential stability of the tracking error is independent of $\eta$. and because the derivative cstimators are $C^{1}$ in $\theta$ and $\Gamma$. wo nocd only assure ourselves that the errors $\left(\theta(0)-\theta_{\times}(0)\right),\left(I^{\prime}(0)-l_{\times}(0)\right)$, and $\left(\xi_{j}^{i}(0)-\theta_{* i}^{(j-1)}(0)\right)$, $i \in \underline{m},\left(j \in \underline{r_{i}}\right)$, are sufficiently small. This is true by hy pothesis.

\section{A Robot Control Example}

The control of robotic manipulators provides a natural selting and motivation for the tracking of implicitly defincd trajectorics. In this section we will apply the controller described above to the problem of output tracking for a simple model of the two-link planar robot arm of Figure 1.

'The links of the robot arm are assumed rigid and of length $l_{1}$ and $l_{2}$. 'T'he minasses of each link are assunned to be point minasses $m_{1}$ and $m_{2}$ localed at the distal cnds of link 1 and link 2 respectively. The actual and desired positions of the end-effector at time $t$ are $x(t)$ and $x_{i t}(t)$ respectively. We wish to make the end-effector (end of the second link) track a prescribed trajectory $x_{t}(l)$ in the Euclidean plane. The configuration-space of the arm is parametcrized by $\theta \in \mathbb{T}^{2}$ where $~^{-2}$ is the two-torus. For our purposes we may view $\mathbb{T}^{2}$ through a single chart from $\mathbb{R}^{2}$ since ncither joint of the arm will cver undergo a full circular 
motion. We will assume that we may exert a control torque at cach joint and will denote the vector of input torques by $\tau \in \mathbb{H}^{2}{ }^{2}$. The forward-kinematics map $\mathcal{F}: \mathbb{E}^{2} \rightarrow \mathbb{R}^{2} ; \theta \mapsto \mathcal{F}(\theta)$ maps the conliguration space to the Euclidean plane. Let $c_{i}:=\cos \left(\theta_{i}\right), c_{i j}:=\cos \left(\theta_{i}+\theta_{j}\right): s_{i}:=\sin \left(\theta_{i}\right)$, and $s_{i j}:=\sin \left(\theta_{i}+\theta_{j}\right)$ with $i, j \in\{1,2\}$. For the two-link arm the forward-kinematics map is

$$
\mathcal{F}(\theta)=\left[\begin{array}{l}
l_{1} c_{1}+l_{2} c_{12} \\
l_{1} s_{1}+l_{2} s_{12}
\end{array}\right] .
$$

The workspace of the robot arm is defined as $\left\{x \in \mathbb{P}^{2}: x=\mathcal{F}(\theta), \theta \in \mathbb{T}^{2}\right\}$, the image of the configuration space through the forward-kinematics map. We choose the output of the system to be $\theta$. We wish to determine a $\tau$ such that the end-effector position $x(t)=\mathcal{F}(\theta(t))$ converges to the desired end-effector position $x_{i l}(t)$.

In this cxample we will use $\theta$ to denote the actual joint angles of the robot arm, $\theta_{*}$ to denote the inverse kinematic solution of $\mathcal{F}(\theta, t)=0$, and $\theta$ to denote the estinnator for $\theta_{x}$.

For cach $x$ in the interior of the workspace, there exist two configurations $\theta$ satisfying $\mathcal{F}(\theta)=x$. Letting $\mathcal{F}^{\prime}(\theta, t):=\mathcal{F}(\theta)-x_{i t}(t)$ the inverse-kinematics problem is to find $\theta_{*}$ satisfying $F(\theta, t)=0$. For robotic manipulators this problem typically has multiple solutions. For certain configurations, kinematics may be inverted by inspection, but in general the problen is difficult and conputationally expensive, making algorithms for inverse-kincmatics an active arca of current research. In the case of our two-link robotic arm, closed form solutions for the inverse kincmatics cxist (sce [Cra89], p.122). For domonstration purposes we will use dynamic inversion to invert the kinematics and we will use the closed form to check our results. As long as $x_{d}$ is kept away from the boundary of the workspace, the two possible inverse kincmatic solutions of $F(\theta, t)=0$ never intersect. We will choose one, by our choice of initial conditions for dynamic inversion, and track it.

The equations of motion for the two link manipulator (see [Cra89], Section 6.8) are

$$
M(0) \ddot{\theta}+V(0, \dot{\theta})+K(0)=\tau
$$

where

$$
\begin{aligned}
& M_{11}(\theta)=l_{2}^{2} m_{22}+2 l_{1} l_{2} m_{2} c_{2}+l_{1}^{2}\left(m_{1}+m_{2}\right) \\
& M_{12}=M_{21}=l_{2}^{2} m_{2}+l_{1} l_{2} m_{2} c_{2} \\
& M_{22}=l_{2}^{2} m_{2}, \\
& V(\theta, \dot{\theta})=\left[\begin{array}{c}
-m_{2} l_{1} l_{2} s_{2} \dot{\theta}_{2}^{2}-2 m_{2} l_{1} l_{2} s_{2} \dot{\theta}_{1} \dot{\theta}_{2} \\
m_{2} l_{1} l_{2} s_{2} \dot{\theta}_{1}^{2}
\end{array}\right],
\end{aligned}
$$

and

$$
K(0)=\left[\begin{array}{c}
m_{2} l_{2} g c_{12}+\left(m_{1}+m_{2}\right) l_{1} g c_{1} \\
m_{2} l_{2} g c_{12}
\end{array}\right] .
$$


The matrix $M(\theta)$ is a positive definite symmetric masis matrix, $V(\theta, \dot{\theta})$ is the vector of centrifugal and Coriolis forces on the manipulator, and $K(\theta)$ is the gravitational force on the arm. The output of our system is $x$ with $x=\mathcal{F}(\theta)$. Let $\theta_{*}(t)$ be the solution of $F(\theta, t)=0$. If we kncw $\theta_{*}(t), \dot{\theta}_{*}(t)$, and $\ddot{\theta}_{*}(t)$ we could sel

$$
\tau=V(\theta, \dot{\theta})+K(\theta)+M(\theta)\left(\ddot{\theta}_{*}-\beta_{1}\left(\dot{\theta}-\dot{\theta}_{*}\right)-\beta_{0}\left(\theta-\theta_{*}\right)\right)
$$

where $\beta_{1}$ and $\beta_{2}$ are positive definite matrices in $\mathbb{E}^{2 \times 2}$ to achieve exponential convergenee of $\theta$ to $\theta_{*}(t)$. But for generality we will assume that we don't know $\theta_{*}$ or its derivatives. We will use dynamic inversion to obtain them.

Wc obtain an cstimator $E^{1}(\Gamma, t)$ for $\dot{\theta}_{x}$ by differentiating $F\left(\theta_{*}, t\right)=0$,

$$
\frac{d}{d l} F\left(\theta_{*} ; t\right)=D \mathcal{F}\left(\theta_{*}\right) \dot{\theta}_{\infty}-\dot{x}_{l}(t)=0
$$

Consequently, $\dot{\theta}_{*}=D \mathcal{F}\left(0_{*}\right)^{-1} \dot{x}_{a}(l)$. Again for generality, rather than symbolically or numerically invert $D \mathcal{F}\left(\theta_{\times}\right)$we will solve $D \mathcal{F}(\theta) \Gamma-I=0$ for $\Gamma$. Thus

$$
E^{-1}(\Gamma, l)=\Gamma \dot{x}_{d}(l) \text {. }
$$

We will also require an estimator for $\ddot{\theta}_{x}$. Note that

$$
\left.\frac{d}{d l} D \mathcal{F}(\hat{\theta})\right|_{\dot{\hat{\theta}}=F^{1}(\Gamma, t)}=\frac{\partial I J \mathcal{F}(\theta)}{\partial 0_{1}} E_{1}^{1}(\Gamma, t)+\frac{\partial D \mathcal{H}(\hat{\theta})}{\partial 0_{2}} E_{2}^{1}(\Gamma, t) .
$$

Differcntiating (26) with respect to t, solving for $\ddot{\theta}_{*}$ : and replacing $D \mathcal{F}\left(\theta_{*}\right)^{-1}$ with $\Gamma$, and $\dot{\theta}_{*}$ with $E^{1}(\hat{\theta}, l)$ gives an estimator for $\ddot{\theta}_{x}$ in terms of $\Gamma$, and $\ell$.

$$
E^{2}(\Gamma, \theta, t):=\Gamma\left(\ddot{x}_{d}-\left.\frac{d}{d l} D \mathcal{F}(\theta)\right|_{\dot{\hat{\theta}}=E^{1}(\Gamma, l)} E^{1}(\Gamma, t)\right) .
$$

For the estimator $E^{M}$ for $\dot{\Gamma}_{*}$ wo get

$$
\left.H^{M}(I, \hat{\theta}, t):=-I^{\prime} \frac{d}{d l} I\right)\left.\mathcal{F}(\hat{\theta})\right|_{\dot{\hat{\theta}}=E^{\prime}(\Gamma, t)} I^{\prime} .
$$

Assembling our results, our controller becomes

$$
\begin{aligned}
\dot{i} & \left.=-\mu l(l) \mathcal{F}(\hat{\theta}) I^{\prime}-l\right)+E^{M}(I, \hat{\theta}, t) \\
\dot{\theta} & =-\mu \Gamma\left(\mathcal{F}(\hat{\theta})-x_{d}(l)\right)+E^{1}(\Gamma, l) \\
\tau & =M(\theta)\left(E^{2}(\Gamma, \hat{\theta}, l)-\beta_{1}\left(\dot{\theta}-E^{1}(\Gamma, l)\right)-\beta_{0}(\theta-\hat{\theta})\right)
\end{aligned}
$$

which, by Theorem 2 give cxponentially convergent tracking $\theta \rightarrow \theta_{*}$.

We choose $x_{d}(t)$ to be a time parameterized figure-eight in the workspace,

$$
x_{d}(t)=[3.75 \cos (\pi t), 2+1.5 \sin (2 \pi t)] .
$$




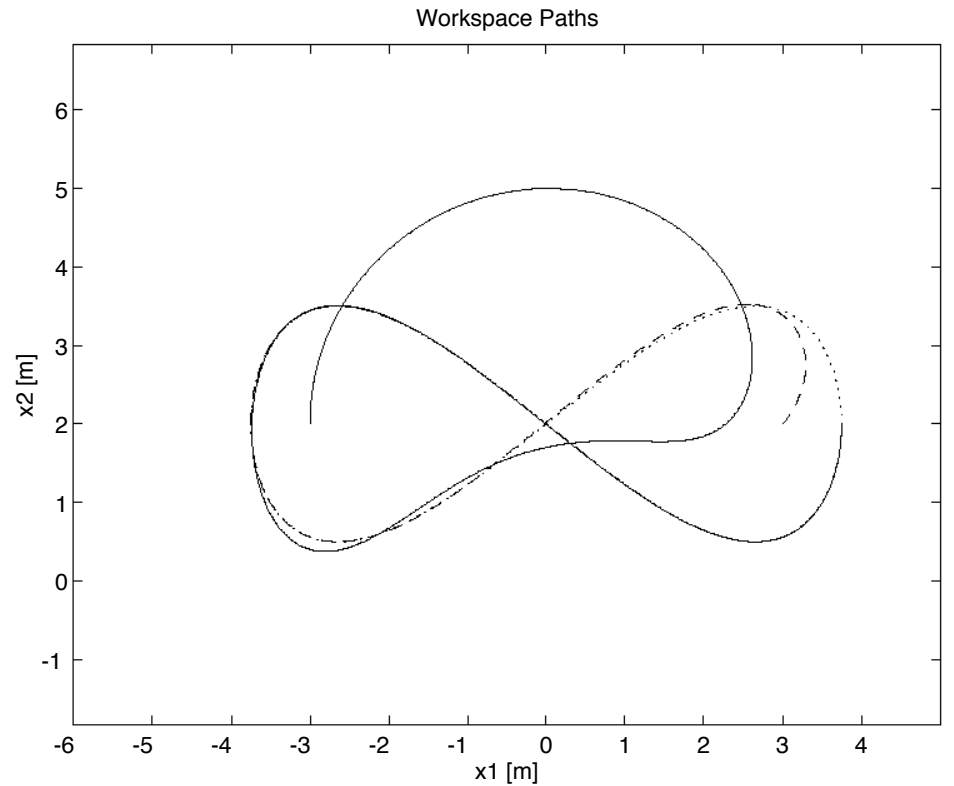

Figure 2: Workspace paths: $\mathcal{F}(\theta)$ (solid), $\mathcal{F}(\hat{\theta})$ (dashed), and $\mathcal{F}\left(\theta_{*}\right)$ (dotted).

Figures 2 through 4 show the results of a simulation. The integration was performed in Matlab [Mat92] using an adaptive step-size Rungc-Kutta intcgrator. The parameters used in the simulation were $\mu=10, \beta_{1}=10 \mathrm{l}$, $\beta_{0}=100 I, l_{1}=3[\mathrm{~m}], l_{2}=2[\mathrm{~m}]$, and $m_{1}=m_{2}=1[\mathrm{~kg}]$ with $g=9.8\left[\mathrm{~m} / \mathrm{s}^{2}\right]$. The initial conditions are $\hat{\theta}(0)=[0, \pi / 2], \Gamma(0)=D \mathcal{F}(\hat{0}(0)=[0,1 / 3,-1 / 2,1 / 3]$, $\theta(0)=[\pi,-\pi / 2], \dot{\theta}(0)=0$ with all angles in radians. Figure 2 shows the resulting cnd-cffector path (solid), desired path (dottcd), and the image of $\theta$ through $\mathcal{F}$ in the workspace (dashed). Both the image of $\theta$ through $\mathcal{F}$, and the path of the end-effector can be seen to converge to the desired path. Figure 3 shows a sinnilar picture, but in configuration space. Again, the convergence of both $\theta$ and $\theta$ to the inverse kincmatic solution corresponding to the desired trajectory can be seen. Figure 1 shows the norm of the estimation error $\hat{\theta}-\theta_{*}$, (top) and the tracking crror $[\theta(t), \dot{\theta}(t)]-\left[\theta_{*}(t), \dot{\theta}_{*}(t)\right]$ (bottom) graphed versus time.

\section{Conclusions}

We have shown that through a well defined dynamical method we may producc explicit cstimators for an implicit output reference trajectory and its time derivatives, where those estimators converge exponentially to the true values of the quantitics which they estimatc. Starting with a statc-fcedback controller 
'Tracking Implicit Trajectories

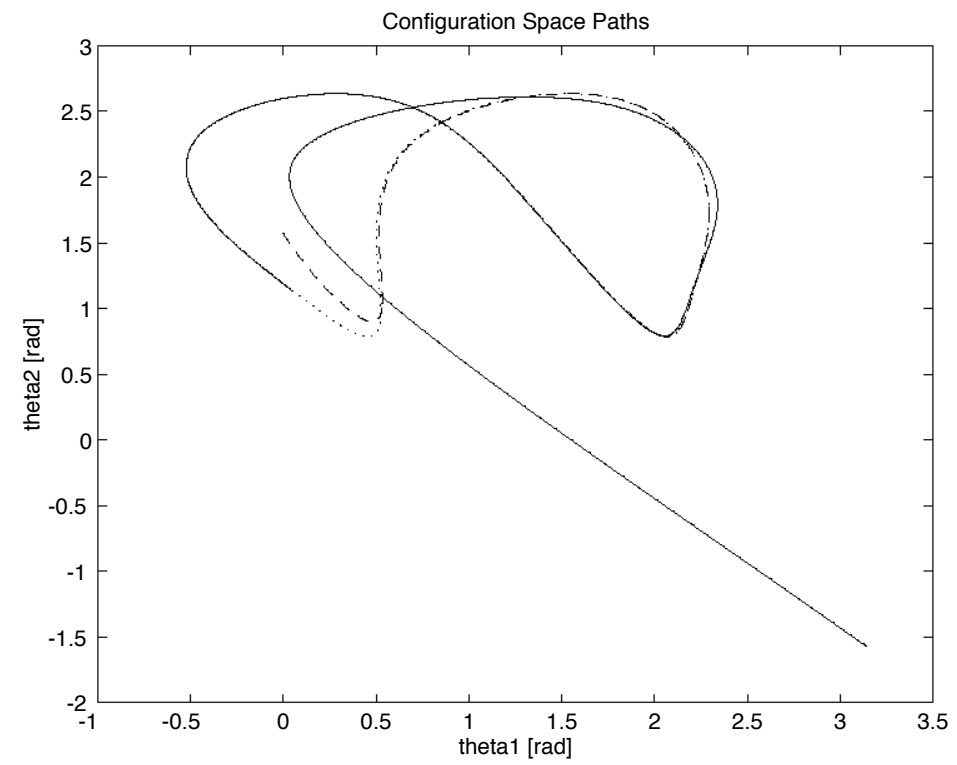

Figure 3: Configuration space paths: $\theta$ (solid), $\theta$ (dashed), and $\theta_{*}$ (dotted).
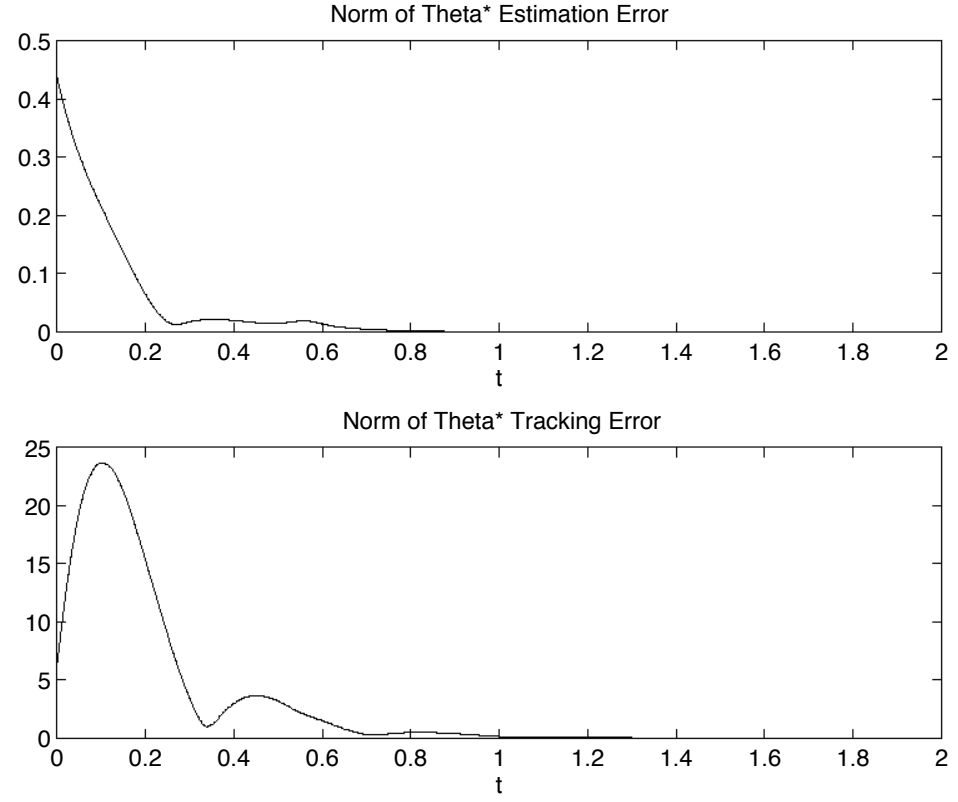

Figure 1: Error norms, $\left\|\ddot{\theta}(l)-0_{*}(l)\right\|_{2}($ top $)$, and $\left\|(0(l), \dot{\theta}(l))-\left(0_{*}(l), \dot{\theta}_{*}(l)\right)\right\|_{2}$ (bottom). 
designed for cxponential tracking of explicit output referenes trajectorics, we replaced the explicit reference trajectory and its time derivatives by our estimators. We then proved, though an appeal to a theorem from the theory of singularly perturbed control systems, that the combination of minimum phase nonlinear plant, dynamic estinator, and controller results in exponentially convergent output tracking with well-bchaved internal dynamics.

The authors are grateful to C.A. Desocr and S.M. Shahruz for their comments and advice.

\section{References}

[Cra89] J.J. Craig. Introduction to Robolics, Mechanics and Conlrol. Addison Woslcy: Now York, sccond cdition, 1989.

[GM94] N. H. Getz and J. H. Marsden. Dynamic inversion of nonlinear maps. Technical Peport 621, Contcr for Pure and Applicd Mathematics, Berkeley, California, 19 December 1994.

[GM95] N. II. Getz and J. E. Marsden. Dynamical methods for polar decomposition and inversion of matrices. Tcchnical Report 624, Conter for Pure and Applied Mathematics, Berkeley, California, 5 January 1995.

[Isi89] A. Isidori. Nonlinear Control Systems, An Introduction. SpringerVorlag, Now York, sccond cdition, 1989.

[Kha92] H.K. Khalil. Nonlinear Systems. Macmillan, New York, 1992.

[Mat92] Mallab. The MatliWorks, Inc., Nalick, Mass., 1992.

[NTV91] S. Nicosia, A. Tornambè, and P. Valigi. A solution to the generalized problem of nonlinear map inversion. Systems and Control letters, $17(5), 1991$. 


\section{A Programs}

In this appendix we include matlats programs used for the simulation of the implicit tracking control of a two-link robot arm. These are the programs that were used to produce the illustrations in the article.

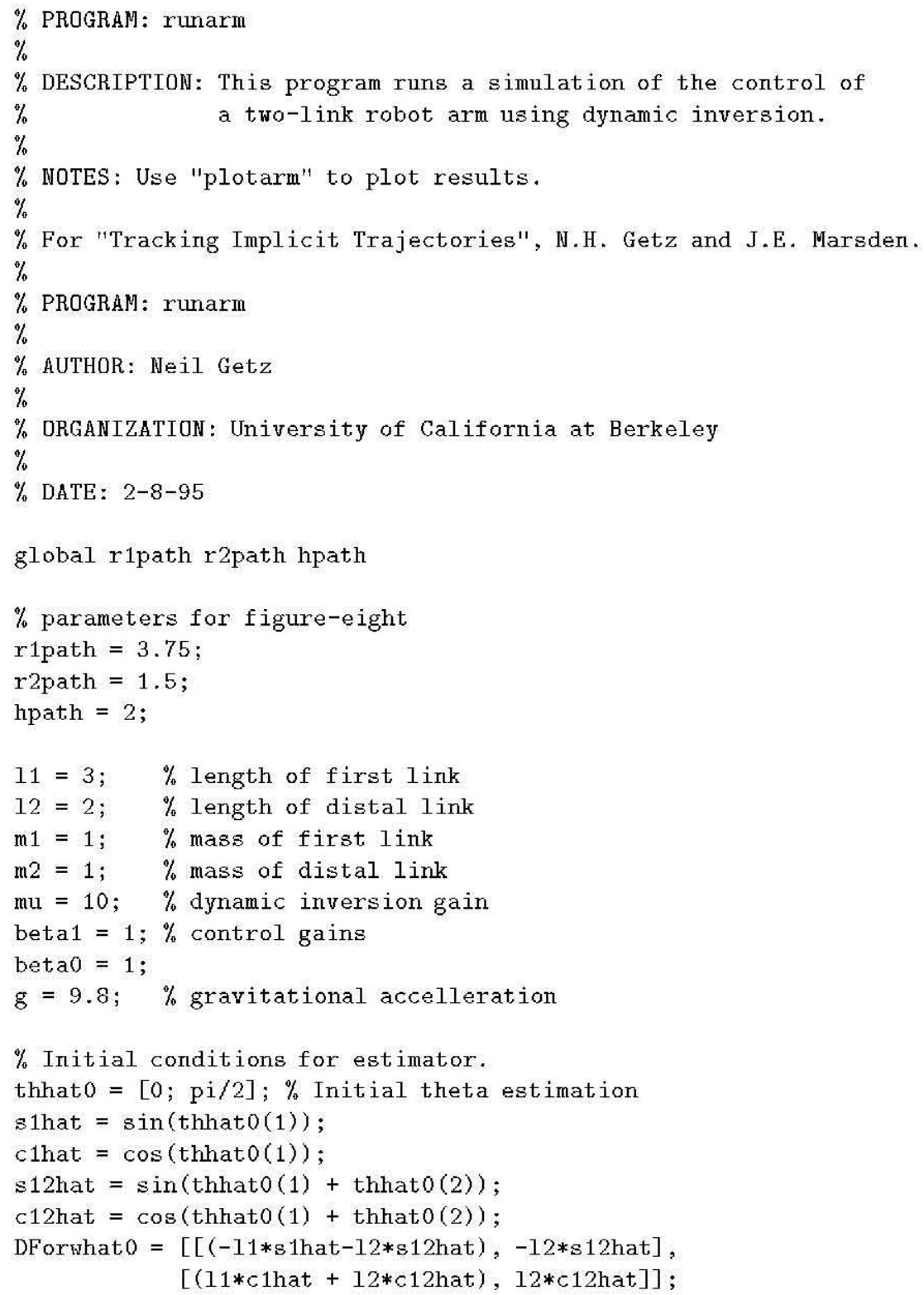




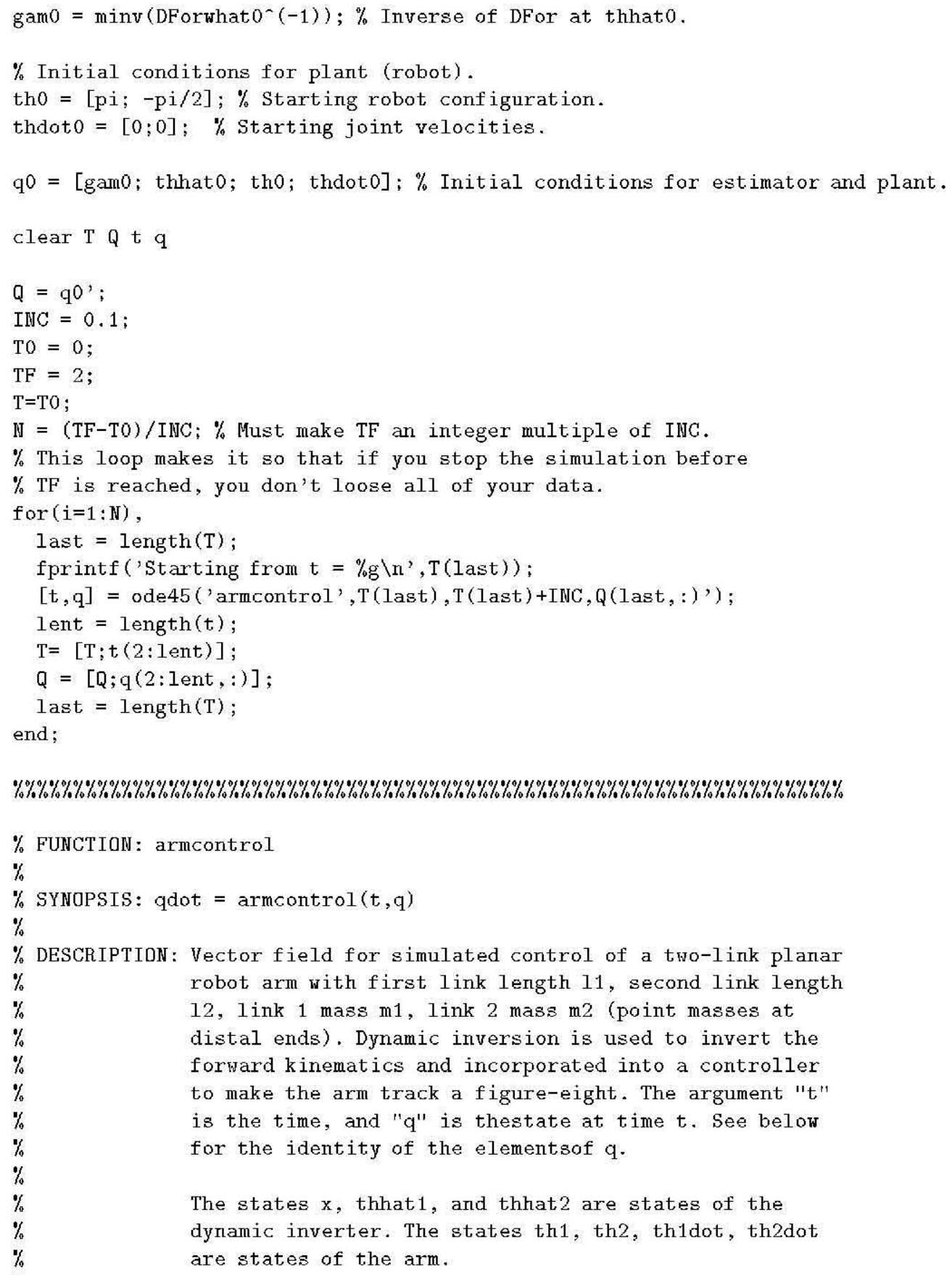




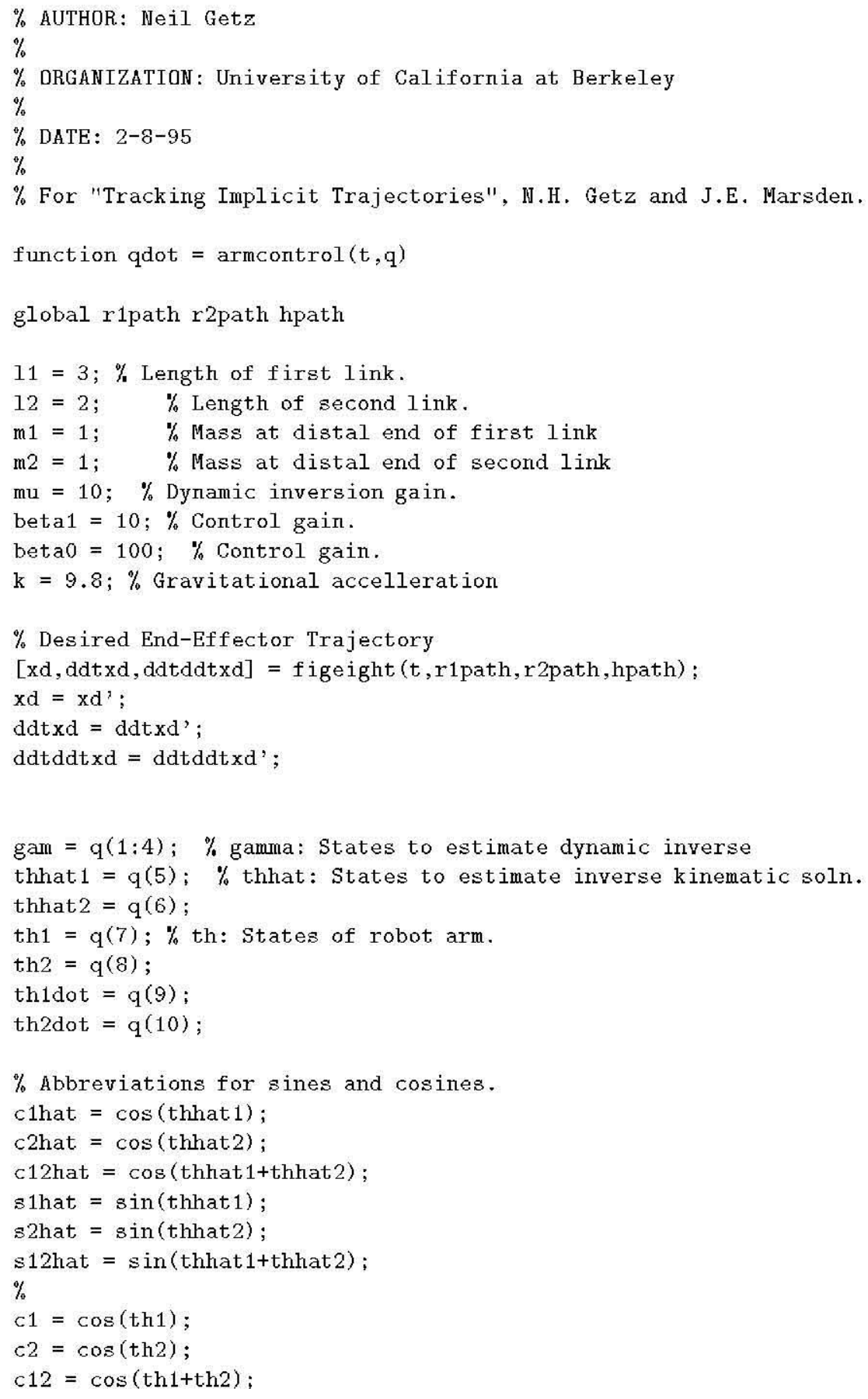




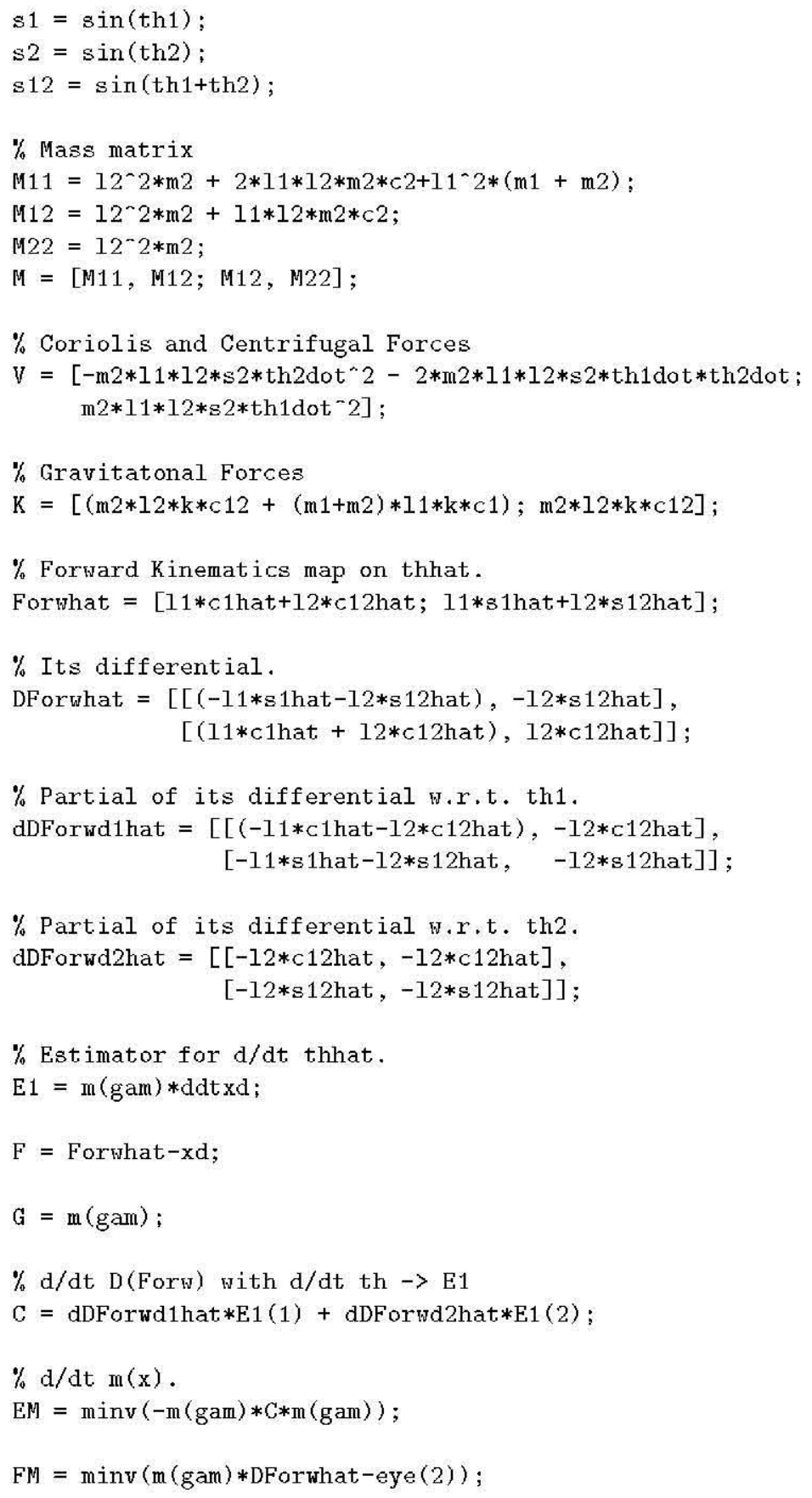




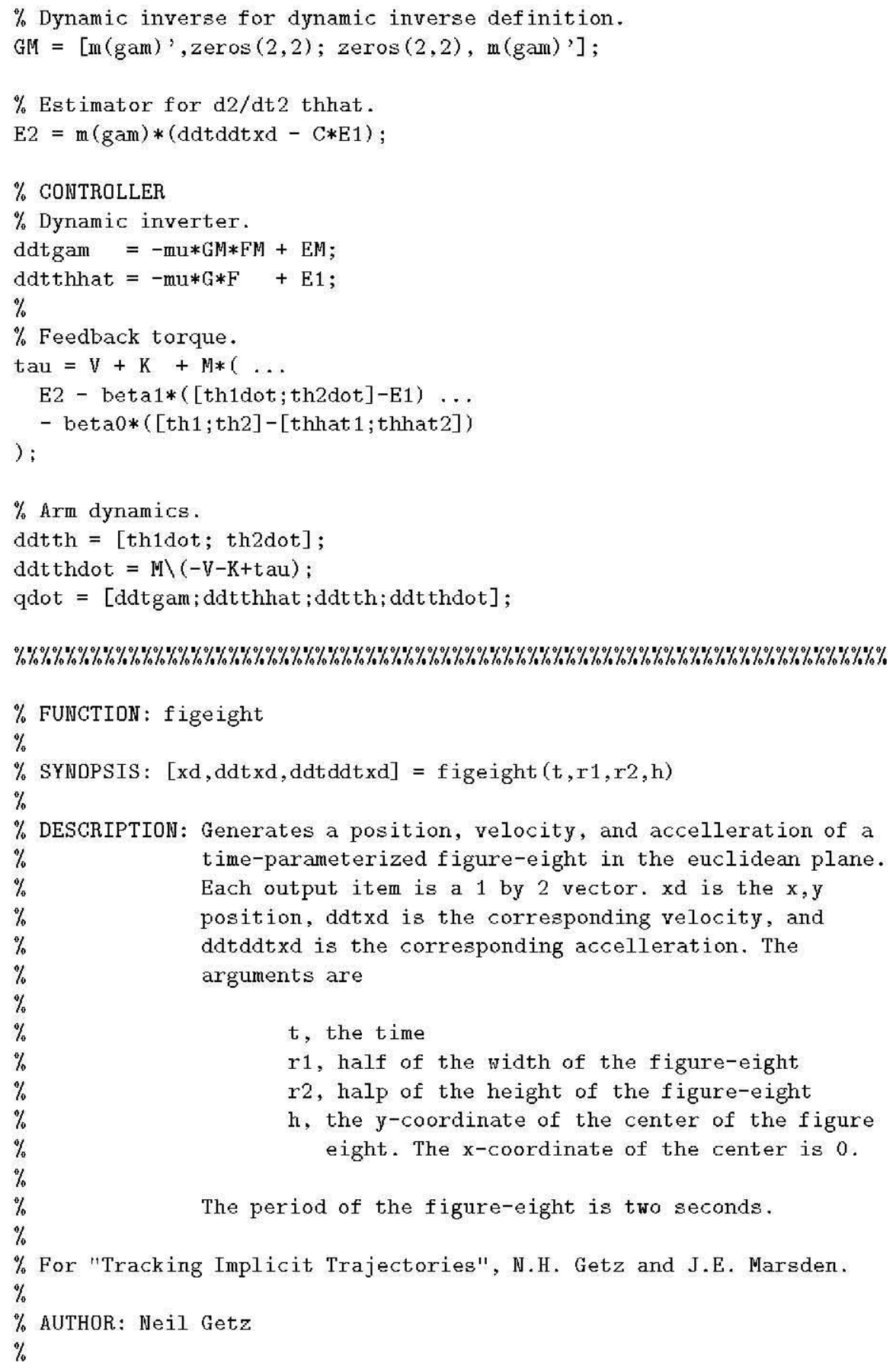




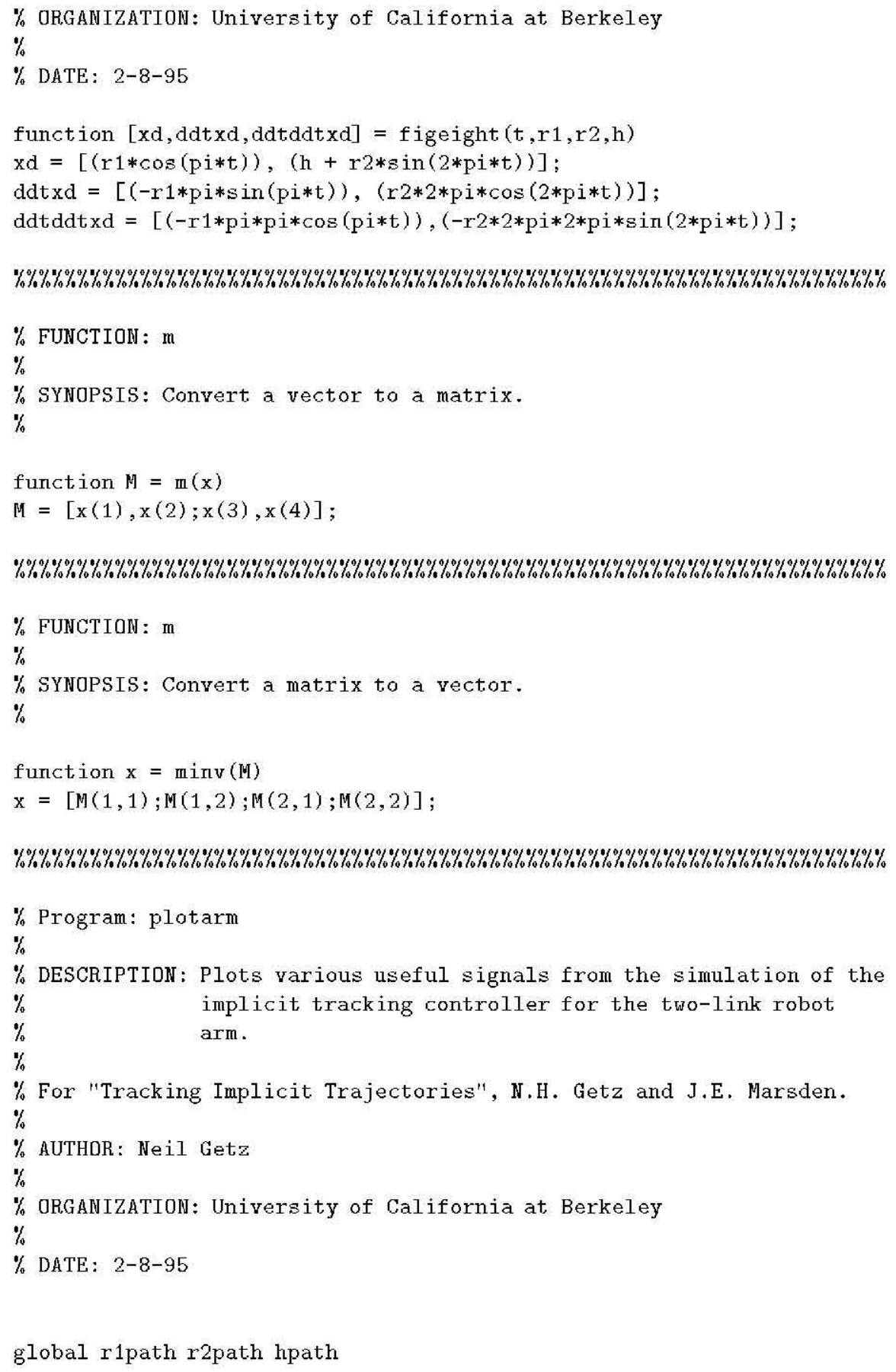




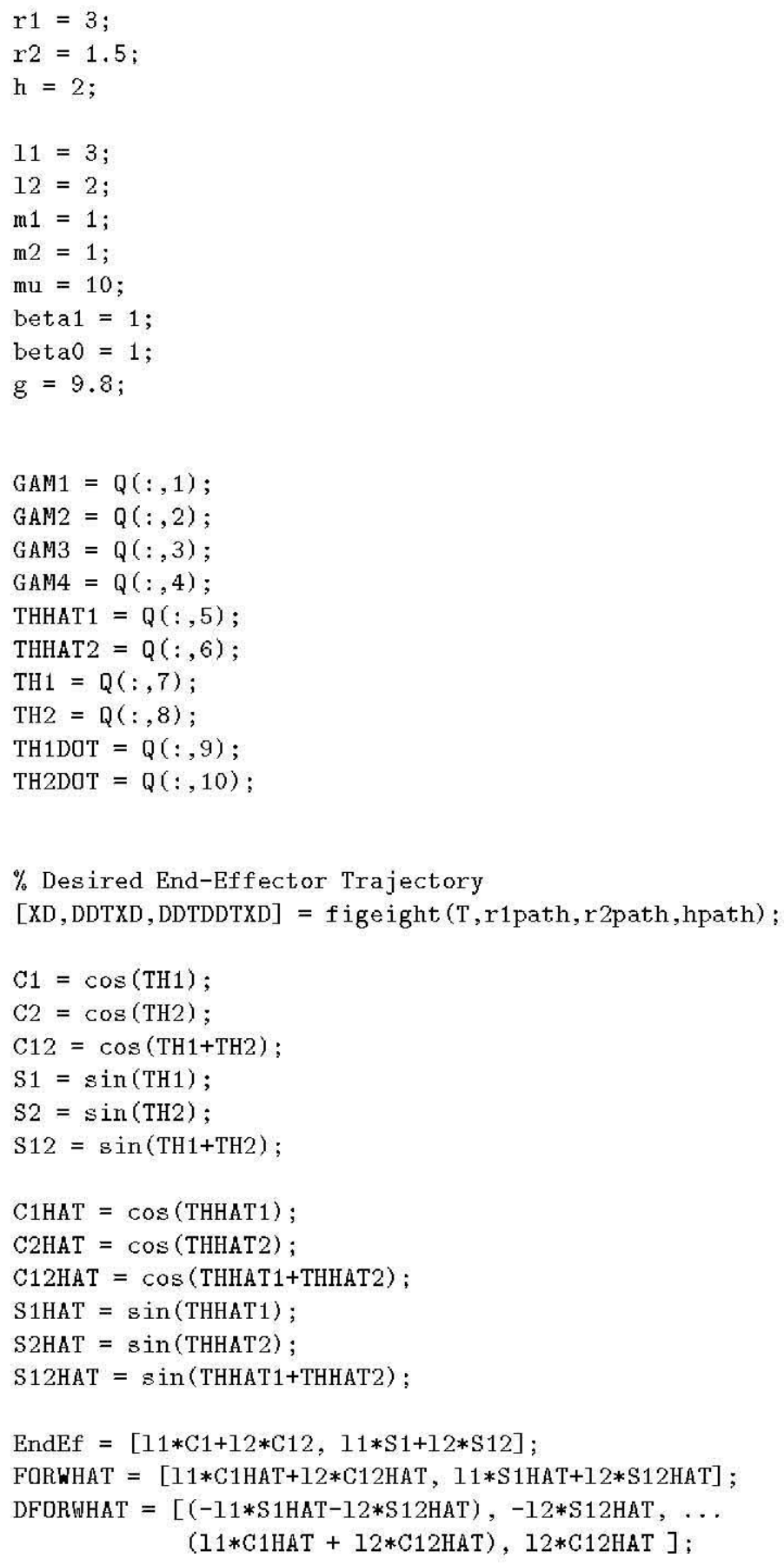




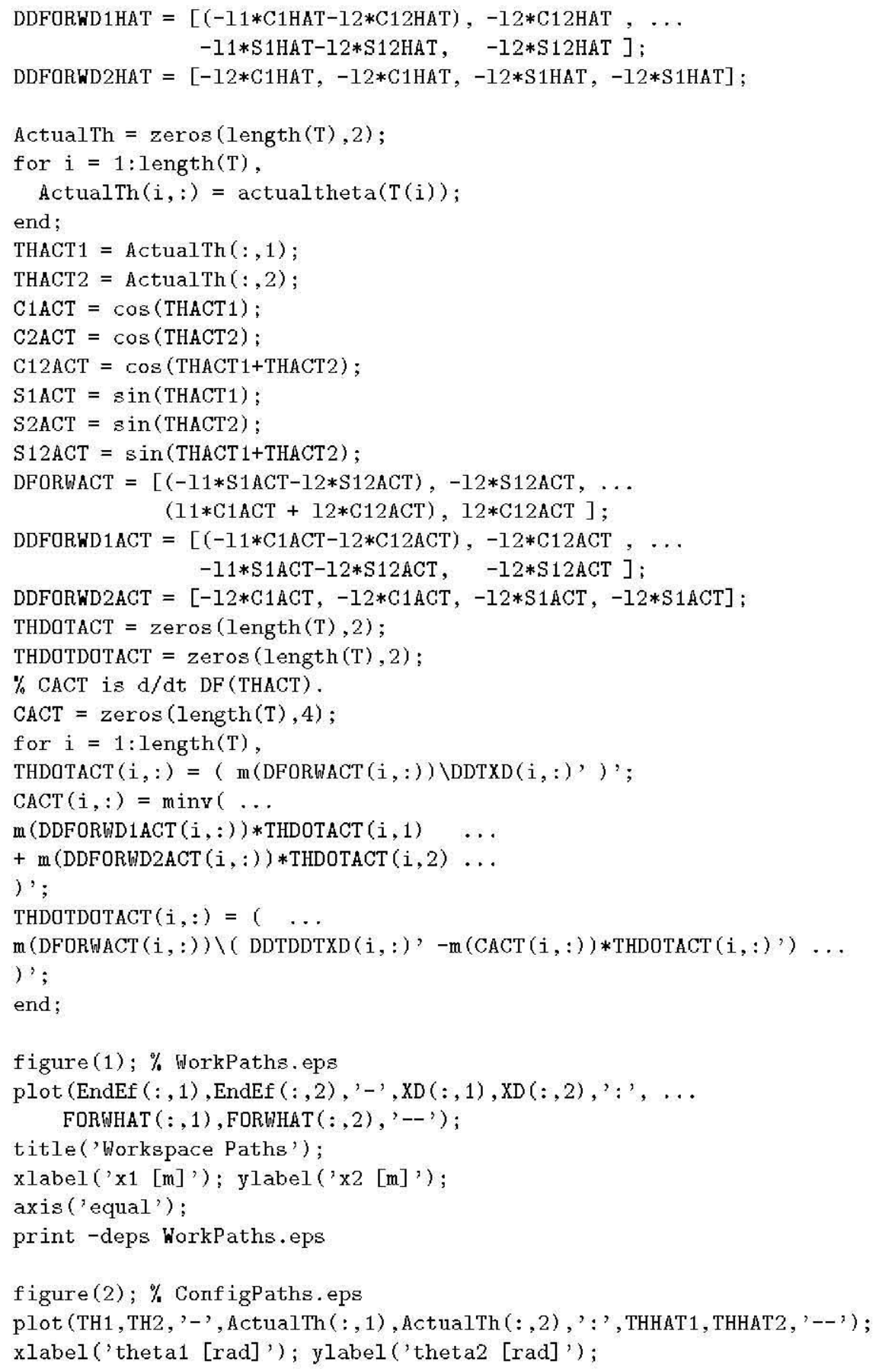




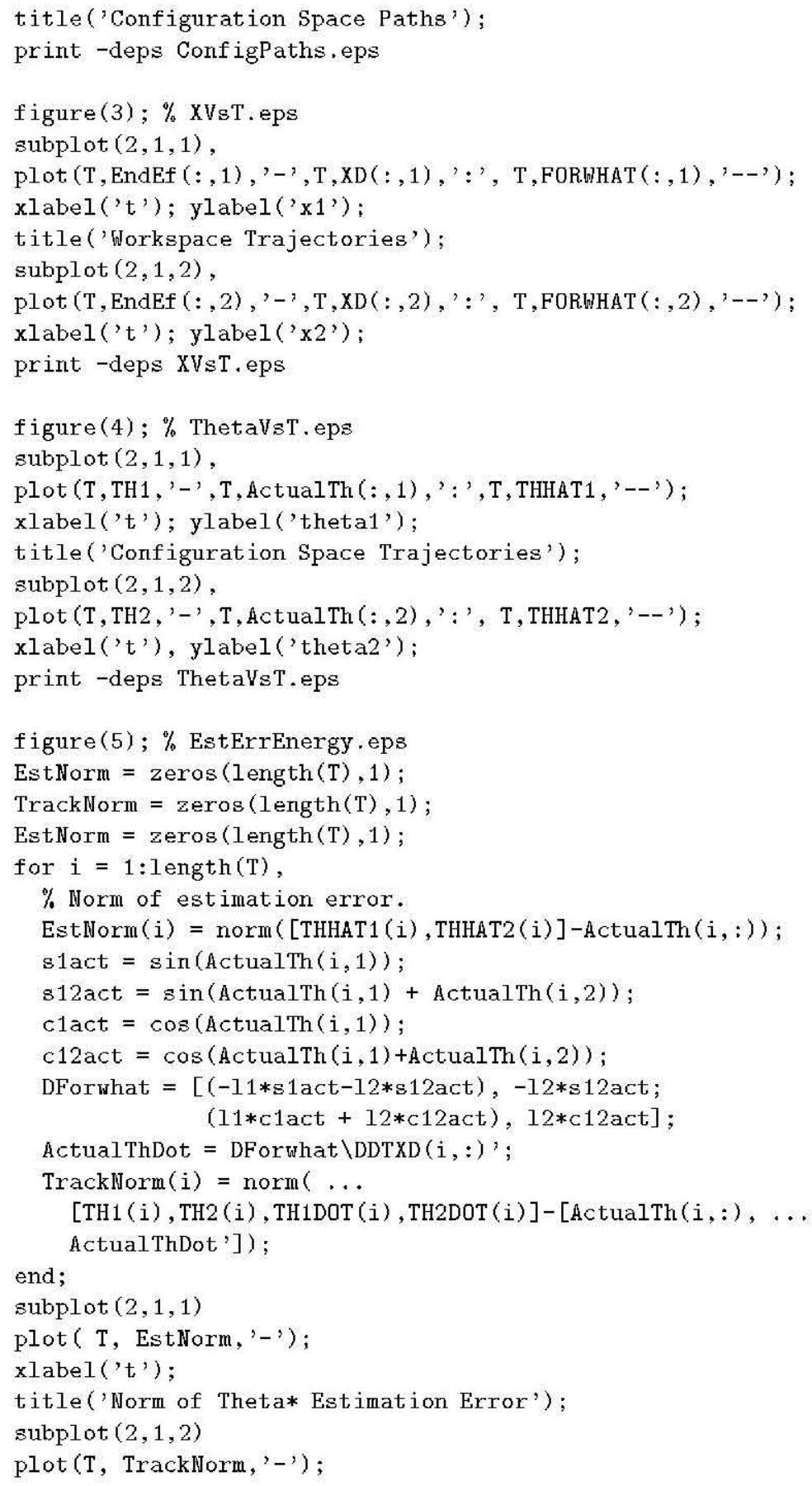




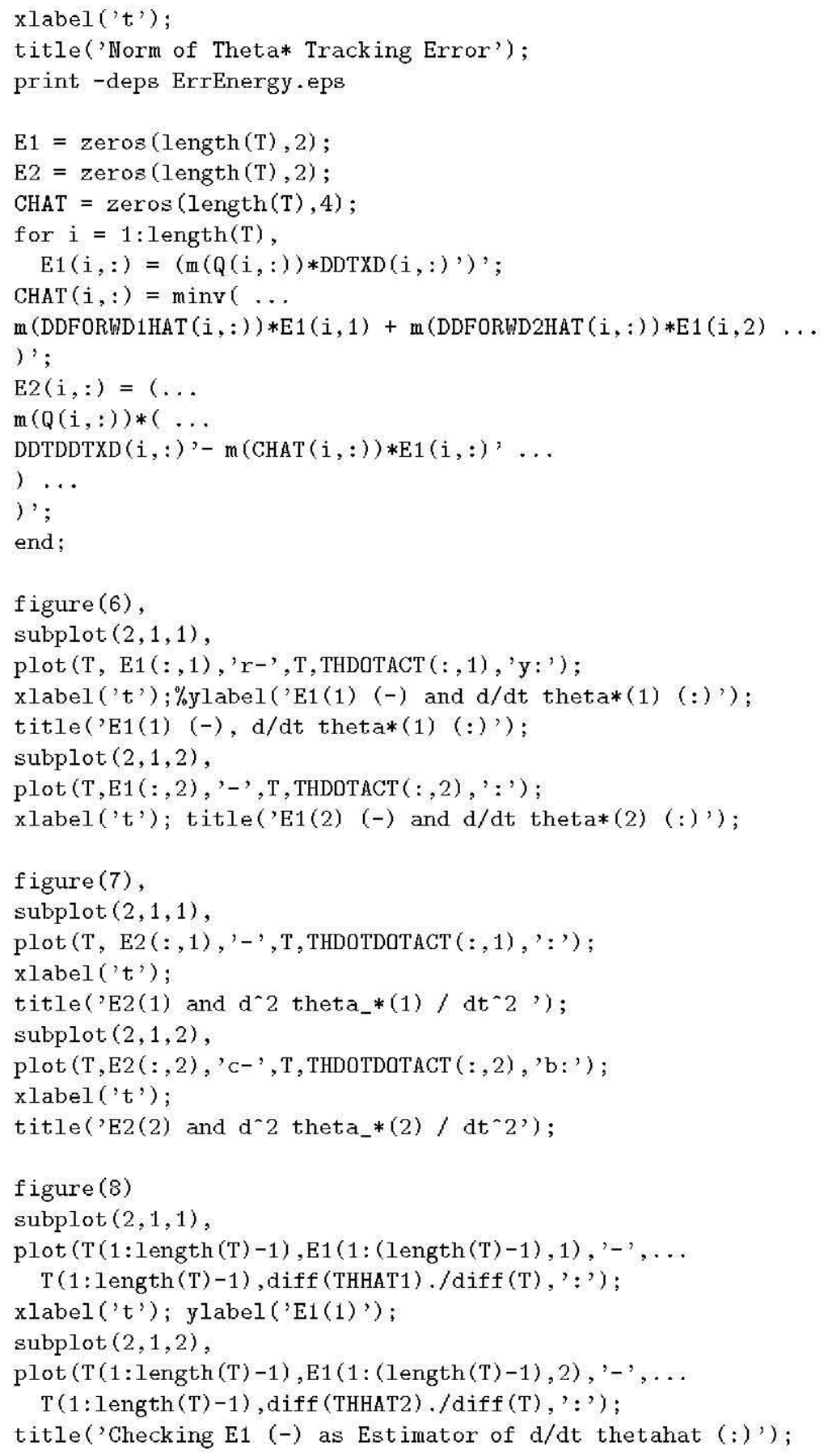


Tracking Implicit Trajectories

xlabel('t'); ylabel('E1(2)'); 